\title{
CLIMATÉRIO: PERSPECTIVAS DE MULHERES PROFISSIONAIS DA SAÚDE DO HOSPITAL UNIVERSITÁRIO NOVA ESPERANÇA, JOÃO PESSOA-PB
}

\author{
*Estela Rodrigues Paiva Alves I \\ Ana Alice Meireles Nóbrega " \\ Adrielly Vidal de Figueiredo Rodrigues" \\ Rebeca Medeiros dos Santos"
}

\section{RESUMO}

O climatério é uma fase natural da vida que normalmente ocorre por volta dos 40 anos de idade, podendo se estender até os 65 . Apresenta-se acompanhado de um conjunto de transformações de natureza biopsicossocial, podendo influenciar consideravelmente as relações interpessoais. Este estudo objetivou compreender os aspectos existenciais da vivência do climatério por mulheres profissionais de saúde e a influência dos sintomas deste período sobre as relações profissionais. Trata-se de um estudo exploratório, descritivo, com abordagem qualitativa de tratamento e análise de dados. Para isso, foram entrevistadas 12 mulheres com idades entre 40 e 65 anos, nos meses de junho e julho de 2019. As mulheres no climatério precoce e histerectomizadas foram excluídas da investigação. Os instrumentos utilizados foram: um roteiro de entrevista semiestruturado com quinze perguntas e o Índice Menopausal de Blatt \& Kuperman (IMBK). Este último foi capaz de identificar e classificar os sintomas do climatério em leve, moderado e intenso. Utilizou-se como método de observação dos dados a análise de conteúdo de Bardin. Este estudo foi aprovado com o CAAE 08331219.5.0000.5179. Após análise, houve criação de duas categorias - os fogachos, ou ondas de calor como fator de risco para depressão e indisposição, ou falta de desejo sexual como expressão da baixa autoestima. Dada a relevância da síndrome do climatério, os sintomas identificados por meio do IMBK e referido pelas mulheres durante as entrevistas, bem como a vivência de cada uma, pode influenciar as relações pessoais no ambiente de trabalho.

PALAVRAS-CHAVE: Saúde da Mulher. Menopausa. Profissionais da Saúde.

Doutora em Enfermagem. Faculdade de Enfermagem Nova Esperança - FACENE. Departamento de Enfermagem. CEP: 58067698, João Pessoa, Paraíba, Brasil.

*Autor correspondente: rodrigues.estela@gmail.com ORCID ID: 0000-0003-3008-2863

Enfermeira. Faculdade Nova Esperança, FACENE. Departamento de Enfermagem. CEP 58067695, João Pessoa, Paraíba, Brasil. Orcid: 0000-0003-4237-5145; 0000-0002-0488-1824; 0000-0003-0338-7768 


\section{INTRODUÇÃO}

O climatério retrata a transição do período reprodutivo para o não reprodutivo, iniciando por volta dos 40 anos e terminando na idade senil em torno dos 65 anos de idade. É um fenômeno endócrino, caracterizado pelo declínio progressivo da produção de hormônios sexuais femininos pelos ovários e culminando com o término definitivo dos ciclos menstruais, evento definido como menopausa que é uma subfase do climatério, reconhecida após 12 meses de amenorreia, ocorrendo no Brasil, em média, entre 48 a 50 anos. ${ }^{1}$

A pré-menopausa é caracterizada pela oscilação dos níveis hormonais afetando a função folicular ovariana, enquanto que a perimenopausa precede a menopausa. A menopausa é identificada após 12 meses do último ciclo menstrual e a pós-menopausa é o período posterior à menopausa, sendo a última etapa que compreende todo o período do climatério. ${ }^{1}$

A mulher no climatério passa por inúmeras alterações biológicas, físicas, psíquicas, sociais e culturais que potencializam o complexo de sinais e sintomas comuns do período, que se manifesta de forma distinta em cada mulher. ${ }^{2}$ Devido a carência do estrógeno, os sinais e sintomas se caracterizam como a síndrome do climatério, podendo prejudicar a saúde. A síndrome compreende distúrbios neurovegetativos, alterações urogenitais e distúrbios metabólicos. Os sintomas se apresentam isoladamente, ou em conjunto, com intensidades variáveis, sendo necessário uma nova adaptação a esta nova fase da vida. ${ }^{1}$

Os distúrbios neurovegetativos incluem os sintomas vasomotores que são os fogachos, ou ondas de calor, acompanhados por sudorese. Outros sintomas são os calafrios, insônia, fadiga, tonturas, ansiedade, cefaleia, irritabilidade, baixa autoestima, modificações nos hábitos sexuais e depressão. A ocorrência da depressão está relacionada, muitas vezes, com o medo de envelhecer e já não atender aos padrões de beleza que a sociedade impõe e, também, por sentimentos de inutilidade relacionados a perda da capacidade reprodutiva. ${ }^{2,1}$

Sob o aspecto geniturinário, o hipoestrogenismo provoca menor capacidade de lubrificação vaginal, causando dispareunia, determinado por dor e desconforto durante a relação sexual. Ocorre diminuição da elasticidade e hipotrofia do tecido genital acarretando o enfraquecimento do assoalho pélvico, causando disfunção urinária (incontinência de esforço), infecções urinárias e a síndrome uretral. ${ }^{1,3}$

Os distúrbios metabólicos são influenciados pelo aumento dos níveis de colesterol e triglicerídeos. Esse quadro pode ser favorável para complicações de doenças cardiovasculares. O metabolismo ósseo também fica reduzido podendo levar a um quadro de osteoporose. ${ }^{1,3}$

A síndrome é dinâmica, transitória ou permanente, tendo prevalência e duração variável e se relaciona com alguns fatores. ${ }^{1}$ Dentre eles, estão os aspectos psicológicos que envolvem a autopercepção da mulher, ou seja, como ela enfrenta esse momento da sua vida; aspectos sociais que se relacionam à interação da mulher com seus familiares e amigos, sendo que estes últimos apresentam forte relação com os aspectos socioculturais, como os mitos, crenças e preconceitos que a sociedade constitui, dissemina e vivencia numa determinada época. ${ }^{4}$

Estudos qualitativos que buscaram compreender ou analisar o significado, percepção ou vivência do climatério pelas mulheres, identificaram que 0 
preconceito social, os conflitos familiares e o desconhecimento da mulher sobre as mudanças dessa fase se constituem como barreiras para viver melhor, feliz e com saúde. ${ }^{5,6,7}$ Outro estudo verificou que a saída dos filhos de casa (ninho vazio) e os conflitos familiares, especialmente com o cônjuge, geravam angústia e sofrimento. ${ }^{4}$

A vivência do climatério sofre diversas influências advindas dos aspectos fisiológicos e do meio sociocultural. Essas transformações e sentimentos podem influenciar as relações interpessoais no trabalho, bem como, no desenvolvimento das atividades laborais. ${ }^{1}$ Buscar entender as dificuldades das mulheres profissionais no climatério poderá revelar novas reflexões, trazendo como contribuição,

\section{MATERIAL E MÉTODOS}

Este é um estudo exploratóriodescritivo de abordagem qualitativa. A coleta ocorreu nos meses de junho e julho de 2019, envolvendo 12 mulheres profissionais da saúde que se encontravam no climatério. A pesquisa foi realizada em uma clínica-escola e no Hospital Universitário Novo Esperança (HUNE), ambos pertencentes às Faculdades de Enfermagem e de Medicina Nova Esperança (FACENE/FAMENE), em João Pessoa/PB, sendo aprovada pelo Comitê de Ética em Pesquisa, sob o protocolo de $n^{\circ}$ 10/2018 e CAAE 08331219.5.0000.5179.

Foram incluídas mulheres com idade entre 40 e 65 anos, faixa etária caracterizada como período do climatério ${ }^{1}$, excluídas aquelas que se recusaram a participar da entrevista e as mulheres histerectomizadas.

Dois instrumentos foram utilizados para coleta de dados. Um roteiro de entrevista semiestruturado, contendo quinze perguntas que foram realizadas, fazendo uso de um gravador de voz, do tipo Mp4, com o propósito uma melhor compreensão sobre o assunto, colaborando para uma intervenção efetiva, melhorando a resposta dos serviços de saúde e ampliando a assistência dos profissionais de saúde. ${ }^{4}$

Com base no exposto, foram elaboradas as seguintes questões norteadoras: Qual a opinião das mulheres profissionais de saúde sobre a vivência do climatério? Como os sintomas, típicos desta fase, podem interferir nas relações pessoais do ambiente de trabalho?

Como objetivo, este estudo buscou compreender os aspectos existenciais da vivência do climatério por mulheres profissionais de saúde e a influência dos sintomas deste período sobre as relações pessoais no ambiente de trabalho.

de alcançar os objetivos traçados e o Índice Menopausal de Blatt \& Kuperman (IBMK). Este último foi validado no Brasil e é capaz de classificar os sintomas do climatério em: leve (escore até 19), moderado (escore entre 20 e 25) e intenso (escore maior que 35$).{ }^{8}$ Quanto maior a pontuação, maior será a intensidade da sintomatologia da mulher e uma pontuação maior que 35 sugere uma aproximação da menopausa, período caracterizado pela ausência da menstruação confirmada após 12 meses. ${ }^{4}$

Os dados sociodemográficos e do perfil de saúde das mulheres foram analisados por meio da estatística descritiva e apresentados em quadros. O material, proveniente dos áudios das entrevistas, foi discutido de acordo com a análise categorial ${ }^{9}$, modalidade temática, nas suas etapas: pré-análise, exploração do material, tratamento dos resultados e interpretação. A pré-análise consistiu na leitura flutuante do conjunto de informações obtidas, após a transcrição das entrevistas. $\mathrm{Na}$ 
exploração do material, buscou-se identificar as unidades de registro e a temática central, recortando os extratos das falas, em cada um dos núcleos de sentidos identificados, o que permitiu a conformação das unidades temáticas.

Na última etapa, ocorreu a análise propriamente dita, por meio do tratamento dos resultados. A interpretação dos dados foi realizada, após uma reflexão crítica dos

\section{RESULTADOS E DISCUSSÃO}

Segundo o IMBK, das 12 mulheres entrevistadas, 6 apresentaram sintomatologia de intensidade leve e as outras 6 , sintomatologia de intensidade moderada, representando $100 \%$ da amostra.

A caracterização sociodemográfica das participantes inclui faixa etária, na qual a maioria (33,3\%) é de 40 a 45 anos. Em relação discursos, com objetivo de desvendar o "conteúdo latente" das participantes e não se limitando apenas ao que foi revelado pelas participantes da pesquisa. Ressalta-se que para preservar a identidade das mulheres estas foram homenageadas com nomes de flores: rosa, lírio, margarida, girassol, amarílis, azaleia, begônia, orquídea, camélia, cravo, crisântemo e dália.

TABELA 1: Características sociodemográficas das mulheres, profissionais de saúde, no climatério. João PessoaPB, jun-jul, 2019.

\begin{tabular}{|c|c|c|}
\hline Faixa etária & Fr. Abs. & \% Fr. Rel. \\
\hline $40-45$ anos & 4 & 33,3 \\
\hline $46-51$ anos & 3 & 25,0 \\
\hline 52-57 anos & 3 & 25,0 \\
\hline $58-65$ anos & 2 & 16,7 \\
\hline Total & 12 & 100 \\
\hline \multicolumn{3}{|l|}{ Escolaridade } \\
\hline Até o ensino médio & 8 & 66,7 \\
\hline Superior incompleto & 1 & 8,3 \\
\hline Superior completo & 1 & 8,3 \\
\hline Pós-graduação & 2 & 16,7 \\
\hline Total & 12 & 100 \\
\hline \multicolumn{3}{|l|}{ Profissão } \\
\hline Psicóloga & 1 & 8,3 \\
\hline Nutricionista & 1 & 8,3 \\
\hline Enfermeira & 1 & 8,3 \\
\hline Técnica de enfermagem & 9 & 75,0 \\
\hline Total & 12 & 100 \\
\hline \multicolumn{3}{|l|}{ Renda familiar } \\
\hline 1-2 salários mínimos & 4 & 33,3 \\
\hline 3-4 salários mínimos & 6 & 50,0 \\
\hline \5 salários mínimos & 2 & 16,7 \\
\hline Total & 12 & 100 \\
\hline \multicolumn{3}{|l|}{ Religião } \\
\hline Católica & 5 & 41,7 \\
\hline Evangélica & 7 & 58,3 \\
\hline Espírita & 0 & 0 \\
\hline Total & 12 & 100 \\
\hline
\end{tabular}


Na Tabela 2, são apresentados o perfil de saúde relacionado ao climatério das mulheres participantes do estudo. Primeiro foi questionado em relação a parceria sexual: $58,3 \%$ delas tinham parceiros e $50 \%$ estavam no período pós-menopausa.

TABELA 2: Perfil de saúde das mulheres, profissionais de saúde, no climatério. João Pessoa-PB, jun-jul, 2019.

\begin{tabular}{|c|c|c|}
\hline Parceria sexual & Fr. Abs. & \% Fr. Rel. \\
\hline Sim & 5 & 41,7 \\
\hline Não & 7 & 58,3 \\
\hline Total & 12 & 100 \\
\hline \multicolumn{3}{|l|}{ Estado menopausal } \\
\hline Pré-menopausa & 1 & 8,3 \\
\hline Perimenopausa & 5 & 41,7 \\
\hline Pós-menopausa & 6 & 50,0 \\
\hline Total & 12 & 100 \\
\hline \multicolumn{3}{|c|}{ Diagnóstico de depressão } \\
\hline Sim & 2 & 16,7 \\
\hline Não & 10 & 83,3 \\
\hline Total & 12 & 100 \\
\hline \multicolumn{3}{|c|}{ Usa antidepressivo/ansiolítico } \\
\hline Sim & 3 & 25,0 \\
\hline Não & 9 & 75,0 \\
\hline Total & 12 & 100 \\
\hline \multicolumn{3}{|c|}{ Faz reposição hormonal } \\
\hline Sim & 2 & 16,7 \\
\hline Não & 10 & 83,3 \\
\hline Total & 12 & 100 \\
\hline \multicolumn{3}{|l|}{ Usa fitoestrógenos } \\
\hline Sim & 3 & 25,0 \\
\hline Não & 9 & 75,0 \\
\hline Total & 12 & 100 \\
\hline
\end{tabular}

No contexto emocional, $16,7 \%$ apresentaram diagnóstico de depressão e $25,5 \%$ faziam uso de antidepressivos. A respeito da reposição hormonal, apenas $16,7 \%$ fazem uso e 25,0\% de fitoestrógenos.

A partir do material proveniente dos áudios das entrevistas, foram criadas duas categorias:

\section{Os fogachos ou ondas de calor como fator de risco para depressão}

O fogacho ou onda de calor é um sintoma vasomotor comum da perimenopausa e pós-menopausa, bastante referido por muitas mulheres nestas fases. ${ }^{10}$ As ondas transitórias se apresentam como um calor intenso na parte superior do tronco, braços e face ou cabeça (tendo início pela nuca), seguido de rubor da face e sudorese profusa, podendo ser acompanhada de palpitações e sensação de ansiedade que duram normalmente de cinco a dez minutos. ${ }^{11,12}$

As ondas de calor raramente ocorrem antes da transição para menopausa, exceto na naquelas mulheres jovens que entram na menopausa induzida devido à ooforectomia (remoção cirúrgica de um ou mais ovários), condições, ou tratamentos médicos que inviabilizem a produção dos hormônios pelos ovários. $^{10,1}$ Podem provocar irritabilidade, insônia, ansiedade e depressão na mulher. ${ }^{13}$ A vulnerabilidade para a depressão aumenta à medida que a produção dos estrógenos diminui1 porque estes esteroides mostram uma nítida ação ansiolítica. ${ }^{12}$ 
Desse modo, mulheres na pósmenopausa tendem a relatar maior nível de ansiedade e depressão. Nesse sentido, Girassol descreve o que sente:

"É um calor horrível. Você fica se abanando e não passa. Você toma muita água e aquela ansiedade vem. Horrível, horrível mesmo!"' (Girassol, 53 anos, pós-menopáusica).

Com base no relato supracitado, Girassol que é técnica de enfermagem, sente-se muito incomodada e irritada com os fogachos. No IMBK, os sintomas apresentados por ela foram classificados como moderados, o que justifica a sua expressão. Para ela, a vivência do climatério é algo negativo, uma vez que deixa claro em seu relato como a sintomatologia afeta a sua vida.

Os níveis de ansiedade e depressão variam. Isto porque dependem de outros fatores que não estão associados ao período do climatério como, por exemplo, mulheres que já apresentaram algum episódio de depressão antes da transição para menopausa e histórico familiar de depressão. ${ }^{13}$ Ao analisar a fala de Begônia, pode-se observar que ela reflete alguns indícios de depressão:

"Tô naquele processo de irritabilidade. Tem dias que eu tô irritada, tem dias que tô chorosa, entendeu? Basta você olhar pra mim de um jeito, que eu já tô chorando [...] (Begônia, 47 anos, perimenopáusica).

Begônia também é técnica de enfermagem e mora sozinha. Apesar de se encontrar na perimenopausa, fase da qual os sintomas tendem a se apresentar de forma mais intensa, sua classificação, segundo o IMBK, foi de sintomatologia leve. Todavia, autores sustentam que a solidão tem sido destacada como sendo fator de risco para depressão, especialmente na fase do climatério. ${ }^{14,15}$

Os episódios de ondas de calor, ou os suores noturnos (quando ocorrem durante o sono) causam um profundo incômodo nas mulheres, por isso são considerados patológicos porque interferem negativamente na qualidade de vida. ${ }^{16,17,18}$ Nota-se, nos depoimentos das técnicas de enfermagem Crisântemo e Azaleia, o sofrimento vivenciado por elas:

"[...] tem os fogachos, os calores. Uma hora você tá com frio, outra hora você tá com calor. Muita dor de cabeça, irritabilidade, dói os ossos [...] (Crisântemo, 43 anos, pósmenopáusica).

Crisântemo, apesar de sua idade (43 anos), já se encontrava na pós-menopausa o que não é comum, pois a menopausa em mulheres brasileiras ocorre normalmente em torno dos 50 anos. $^{1}$ Com manifestações do climatério de intensidade moderada, a entrevistada tem diagnóstico médico de depressão, faz uso de antidepressivos e ansiolíticos, uso de fitoestrógenos, substâncias naturais, produzidas pelas plantas que apresentam uma estrutura química diferente dos estrógenos, mas que atuam da mesma maneira. Dois estudos de revisão apontam os benefícios e controvérsias do seu uso na redução dos sintomas do climatério e opções terapêuticas fitoterápicas, respectivamente. ${ }^{19,20}$

Antidepressivos, ansiolíticos e a Terapia de Reposição Hormonal, também são capazes de reduzir a intensidade dos sintomas do climatério. ${ }^{12}$

“É horrível essa questão do calor. Ele é insuportável! Não tem ventilador, nem água e nem vento. Eu sou sincera de dizer a você, é um inferno!" (Azaleia, 41 anos, perimenopáusica).

Azaleia também teve a intensidade de seus sintomas do climatério classificados como moderado. Diferente da depoente anterior, se encontrava na perimenopausa o que é 
esperado para a sua idade. A entrevistada demonstra total desconforto nessa fase, diante da sintomatologia apresentada.

Graus de ansiedade, irritabilidade e depressão são os sintomas que mais levam as mulheres no climatério a procurarem por ajuda médica especializada. Há uma relação direta entre a severidade dos sintomas do climatério e da depressão, ou seja, quanto maior a intensidade dos sintomas do climatério, maiores e mais severos serão os sintomas depressivos. ${ }^{21}$

\section{Indisposição ou falta de desejo sexual como expressão da baixa autoestima}

Muitas mulheres, ao expressarem suas queixas nos consultórios especializados, relatam também baixa autoestima, medo e sentimentos negativos sobre sua aparência física. Nestes casos, a diminuição da libido não só tem relação com o hipoestrogenismo, mas também, com a visão que a mulher tem de si mesma. ${ }^{22}$

\begin{abstract}
"Não tenho vontade de fazer sexo, demora para chegar vontade. Me sinto muito acabada, muito pra baixo." (Cravo, 41 anos, pré-menopáusica).
\end{abstract}

Cravo é psicóloga e ainda apresenta ciclo menstrual regular. Faz uso de psicofármacos, muito embora na entrevista, não tenha mencionado ter recebido diagnóstico médico para ansiedade ou depressão. Segundo o IMBK, a sintomatologia de Cravo foi classificada como leve. No seu relato, fica claro que ela apresenta uma percepção negativa da sua autoimagem e consequente baixa autoestima.

Remédios que aumentam a quantidade de serotonina no cérebro, como é o caso dos antidepressivos e ansiolíticos, possuem maior potencial para reduzir o desejo sexual. ${ }^{23}$ Somam-se a isto, a grande dificuldade que algumas mulheres têm de aceitar o envelhecimento físico, pois em nossa cultura existe uma supervalorização do corpo jovem. Sentir-se "acabada", como referiu Cravo, é uma expressão de não aceitação. Por esse motivo, algumas mulheres acabam tendo sua autoestima reduzida, com o passar dos anos, porque não conseguem mais acompanhar o alto padrão de beleza exigido. Desse modo, a baixa autoestima pode prejudicar o relacionamento da mulher com o seu parceiro e afetar a sua vida sexual. ${ }^{24}$

\begin{abstract}
"Na vida sexual também abala né? O marido começa a perguntar assim: o que é que está havendo? Não gosta mais de mim? Tem outro? Aí deixa a gente mais pra baixo ainda né? Poxa, o marido desconfiando da gente!" (Margarida, 62 anos, pós-menopáusica).
\end{abstract}

Margarida é nutricionista e já está na menopausa. Em sua entrevista referiu ter sido diagnosticada com ansiedade e depressão. Faz tratamento com psicofármacos, além da reposição hormonal e uso de fitoestrógenos.

É comum que a diminuição do desejo sexual ocorra nos períodos de peri e pós menopausa. Nesses casos, a terapia estrogênica (reposição hormonal) pode ser uma grande aliada, especialmente nos casos em que existam a presença de outros sintomas associados como alterações do sono e do humor, respeitando as contraindicações absolutas, como o uso em mulheres com câncer de mama, câncer de endométrio, tromboembolismo agudo, hepatopatia aguda e/ou grave, diabetes com lesão de órgãoalvo, porfiria e sangramento uterino sem causa diagnosticada. ${ }^{12,25}$ Os fitoestrógenos são um outro recurso utilizado quando há contraindicação da Terapia de Reposição 
Hormonal, podendo também serem usados em combinação. ${ }^{19}$

\begin{abstract}
"Não tenho vontade de fazer sexo, demora para chegar vontade. Me sinto muito acabada, muito pra baixo." (Cravo, 41 anos, pré-menopáusica).
\end{abstract}

Mulheres fragilizadas pela não aceitação do próprio corpo envelhecido não se sentem à vontade para se relacionar sexualmente com o parceiro. Além disso, deve serconsideradaaqualidadedorelacionamento, pois nem todos os companheiros estão preparados para compreender e ajudar as mulheres diante de inúmeras alterações que ocorrem no período do climatério. ${ }^{26}$ A alteração mais relatada e percebida pelas mulheres é a falta de lubrificação vaginal que poderá causar desconforto durante $\mathrm{o}$ ato sexual, angústia e irritabilidade, interferindo na qualidade de vida da mulher. ${ }^{22}$

A vivência da sexualidade para algumas mulheres nem sempre é vista como fonte de sofrimento, como evidenciado nas falas de Lírio, Girassol e Margarida:

\footnotetext{
"Ainda tenho a libido, tenho prazer demais. Sou meio fogosa! A gente foi muito aberto um com o outro e a gente vive em plena paz até hoje em 32 anos de casado [...] Eu me acho linda, bela, maravilhosa, charmosa, elegante. Gordura pra mim não é nada, às vezes me chamam de gordinha, mas pode chamar que eu sou assumida mesmo! [...] Pra que que eu vou me preocupar se meu marido me ama do jeito que eu sou. (Lírio, 60 anos, pós-menopáusica).

"Minha libido não diminui. [...] Eu me acho linda, maravilhosa." (Girassol, 53 anos, pós-menopáusica).

"Hoje eu já gosto de me ver no espelho, sei que o corpo já está assim pela idade." (Margarida, 62 anos, pósmenopáusica).
}

À medida que as mulheres entram no período do climatério, outras fontes de estrogênio aumentam sua síntese, tornandose influentes no organismo. A síntese de estrogênio, situada fora dos ovários, foi descoberta em 1974, quando foi observado no tecido adiposo que os andrógenos foram convertidos em estrógenos, desde então várias outras fontes foram descobertas. 0 estrogênio pode ser produzido no cérebro, ossos, fígado, glândula adrenal, pele, vaso sanguíneo e tecido adiposo, sendo essa última considerada a principal fonte de estrogênio depois das gônadas feminina e masculina. Entretanto, a síntese de estrogênio só é possível desde que haja a presença da aromatase (proteína responsável pela síntese do estrógeno). ${ }^{27}$

A sexualidade vai muito além de processos hormonais, é um fenômeno complexo que envolve vários fatores como: a intimidade, boa comunicação e fantasias do casal que, em conjunto, é responsável por obter o desejo, prazer e satisfação sexual. Nesse sentido, estudo revela que o bom relacionamento com o companheiro tornará a vida sexual melhor ${ }^{4}$, potencializando a fala de Lírio. Quando as mulheres podem contar com o apoio, compreensão e ajuda de seus familiares, amigos e, especialmente dos seus parceiros, a jornada pelo climatério se torna mais tranquila e, consequentemente, melhora a qualidade de vida. ${ }^{4,26}$

Outro ponto importante, representado nas falas de Girassol e Margarida, e que contribui positivamente para a sexualidade feminina neste período, está ligado a subjetividade da mulher. Ou seja, boa autoestima e autovalorização, pois quando a mulher está satisfeita com sua aparência física e é autoconfiante, ela se sente segura no desempenho da sua sexualidade. Pode-se dizer então que o climatério significa uma etapa diferente para cada mulher, ou seja, nem todas as mulheres 
sofrerão com a síndrome do climatério. ${ }^{24,28}$

Os fogachos podem ser intensificados pelo uso do jaleco ou fardamento. Quando associados à insônia, tontura, dores, ansiedade e irritabilidade, interferem na jornada de trabalho das mulheres, diminuindo sua produtividade. Um estudo quantitativo, realizado com 385 mulheres trabalhadoras, mostrou que a síndrome do climatério é o que

\section{CONCLUSÃO}

Muitas são as mudanças que as mulheres vivenciam na fase do climatério. Destacando que alterações psicológicas ou fisiológicas e a existência de sinais e sintomas característicos dessa fase afetam a qualidade de vida.

O estudo permitiu compreender que nem todas apresentam a mesma sintomatologia. Apesar da maioria descrever os mesmos sintomas, em algumas se manifestam de forma distinta. De modo geral, de acordo com o IMBK, as participantes apresentaram uma intensidade de sintomatologia de leve a moderada. Os sintomas predominantes foram os fogachos, tristeza, artralgia/mialgia, insônia e cefaleia.

As mulheres são acometidas com sentimentos de baixa autoestima, ansiedade e problemas relacionados a depressão e alterações no padrão sexual como a perda da libido.

A síndrome do climatério influencia negativamente as relações pessoais no mais afeta a mulher no ambiente de trabalho. ${ }^{29}$

Outro ponto importante, algo também relevante e que pode repercutir negativamente no trabalho, é a falta de confiança em si e a baixa autoestima. A sensação de incapacidade, medo e a dificuldade de se aceitar e se conhecer pode atrapalhar as relações interpessoais, dificultando seu crescimento profissional. ${ }^{29}$

ambiente de trabalho de algumas mulheres. Os sintomas identificados, por meio do IMBK, e referidos pelas mulheres, durante as entrevistas, bem como a vivência de cada uma, podem influenciar nas relações pessoais de algumas mulheres no ambiente de trabalho.

Portanto, é necessário que a assistência à saúde da mulher no climatério leve em consideração não só os aspectos físicos desta fase, mas também, os aspectos psicológicos e socioculturais da mulher. Vale ressaltar que a educação em saúde é imprescindível, sendo necessário desenvolver ações junto às mulheres com o intuito de conhecer o funcionamento de seu corpo. É necessária a atualização dos profissionais de saúde para melhor assistirem às mulheres nesse período.

Recomenda-se o desenvolvimento de novos estudos sobre o impacto do climatério no ambiente de trabalho, utilizando populações maiores e outras metodologias, visto que a atual pesquisa trabalhou com uma amostra pequena, sendo esta a limitação do estudo. 


\title{
CLIMACTERIC PERIOD: PERSPECTIVES OF HEALTH PROFESSIONAL WOMEN AT THE NOVA ESPERANÇA UNIVERSITY HOSPITAL, JOÃO PESSOA-PB
}

\begin{abstract}
The climacteric period is a natural phase of life that normally occurs around the age of 40, and can extend to 65. It can be accompanied by a set of biopsychosocial transformations, which can considerably influence interpersonal relationships. This study aimed to understand the existential aspects of the climacteric experience by women health professionals and the influence of the symptoms of this period on professional relationships. This is an exploratory, descriptive study, with a qualitative approach to data treatment and analysis. For this, 12 women aged between 40 and 65 years were interviewed, in June and July 2019. Women in the early climacteric period and hysterectomized were excluded from the investigation. The instruments used were: a semi-structured interview script with fifteen questions and the Blatt \& Kuperman Menopausal Index (BKMI). The latter was able to identify and classify climacteric period symptoms as mild, moderate and intense. Bardin's content analysis was used as the data observation method. This study was approved with CAAE 08331219.5.0000.5179. After analysis, two categories were created - hot flushes or hot flashes as a risk factor for depression and indisposition or lack of sexual desire as an expression of low self-esteem. Given the relevance of the climacteric syndrome, the symptoms identified through the BKMI and referred by women during the interviews, as well as the experience of each one, can influence personal relationships in the work environment.
\end{abstract}

KEYWORDS: Women's Health. Menopause. Health professionals.

\section{REFERÊNCIAS}

1. Ministério da Saúde (BR). Manual de Atenção à Mulher no Climatério/Menopausa. Brasília: Ministério da Saúde, 2008.

2. Alcântara DS, Lemos RB, Suzane H, Marinho G, Teixeira ACS. A vivência do climatério por mulheres atendidas em uma unidade básica de saúde no município de Gurupi-TO. Rev Amazônia. 2018; $12(1):$ 43-7.

3. Melo CRM, Reis ES, Silva LCFP, Sola EPS, Chofakian CBN. Aplicação do índice menopausal de Kupperman: um estudo transversal com mulheres climatéricas. Rev. Saúde Pública do Paraná. 2016; 17(2): 41-50.

4. Alves ERP. Terapia Comunitária Integrativa e mulheres vivenciando o climatério: uma pesquisa ação-intervenção. 2017. 178f. [Tese]. João Pessoa: Universidade Federal da Paraíba; 2017.

5. Cardoso MR, Camargo MJG. Percepções sobre as mudanças nas atividades cotidianas e nos papéis ocupacionais de mulheres no climatério. Cad. Ter. Ocup. UFSCar. 2015; 3(23): 553-69.

6. Souza SS, Santos RL, Santos ADF, Barbosa MO, Lemos CS et al. Mulher e climatério: concepções de usuárias de uma unidade básica de saúde. Reprod. Clim. 2016; 2(32): 87-9.

7. Zanotelli SS, Ressel LB, Borges ZN, Junges CF, Sanfelice C. Vivências de mulheres acerca do 
climatério em uma unidade de saúde da família. Pesq.: cuid. fundam. Online. 2012; 1(4): 2800-11.

8. Sousa RL, Sousa ESS, Silva JCB, Filizola RG. Fidedignidade do teste-reteste na aplicação do índice menopausal de Blatt e Kupperman. Rev. Bras. Ginecol. Obstet. 2000; 22(8): 481-7.

9. Bardin L. Análise de conteúdo. São Paulo: Brasil; 2011.

10. Santoro N, Epperson CN, Mathews SB. Menopausal Symptoms and Their Management. Endocrinol Metab Clin North Am. 2015; 3(44): 497-515.

11. Kronenber F, Downey JA. Thermoregulatory physiology of menopausal hot flashes: a review. Can J Physiol Pharmacol. 1987; 2(65): 12-24.

12. Fonseca AM, Bagnoli VR, Arie WMY, Fassolas G, Cezarino PYA, Baracat EC. Terapia estrogênica no climatério: qual a melhor via? Uma resposta baseada nas melhores evidências. FEMINA. 2010; 38(2): 89-100.

13. Lomônaco C, Tomaz RAF, Ramos MTO. O impacto da menopausa nas relações e nos papeis sociais estabelecidos na família e no trabalho. SBRH. 2015; 2(30): 58-66.

14. Bodner E, Bergmam YS. Solidão e sintomas depressivos entre idosos: o papel moderador da expectativa subjetiva de vida. Psiquiatria Res. 2016; 3(22): 78-82.

15. Santos EJP, Araújo IFF, Alves ERP. O paradoxo do ninho vazio: relatos de mulheres no climatério em rodas de terapia comunitária. João Pessoa. 2018; 1(2): 1-9.

16. Santos TR, Pereira SVM, Santos LS. Intensidade da sintomatologia climatérica em mulheres pós menopausa. Rev Rene. 2016; 2(17): 225-32.
17. Hoffman S, Bradshaw, Halvorson, Schaffer AND CORTON. Williams Gynecology 3rd McGraw-Hill Education. 2016; 3 (2): 8-10.

18. Martins MA, Nahas EA, Neto J, Buttros DA, Traiman P. Quality of life in postmenopausal women, users and non-users of hormone therapy. Rev Bras Ginecol Obstet. 2009; 4(31): 196-202.

19. Souza EL, Monyka BLS. Vantagens do uso de fitoestrógenos no tratamento de reposição hormonal: revisão de literatura Reon Facema. 2018; 4(4):1324-29.

20. Rocha BAM, Pereira VSM, Correia JQ. Terapias complementares: fitoterapia como opção terapêutica no climatério e menopausa. Rev. de Ciênc. da Saúde Nova Esperança. 2018; 1(16): 1-9.

21. Wang HL, Booth AC, Tang SM, Chen $\mathrm{CH}$. Depressive symptoms in Taiwanese women during the peri- and post-menopause years: Associations with demographic, health, and psychosocial characteristics. Maturitas. 2013; 75(4):355-60.

22. Fonseca, FM, Santos FF, Costa FM, Santos JAD, Carneiro JA. Climatério: influência na sexualidade feminina. Rev. UNINCOR. 2015; 13(2): 639-48.

23. Cordás TA, Laranjeiras M. Efeitos colaterais dos psicofármacos na esfera sexual. Rev. Psip. Clín. 2006; 33(3): 168-73.

24. Freitas ER, Barbosa AJG. Qualidade de vida e bem-estar psicológico no climatério. Arq. bras. psicol.. 2015; 67(3): 112-24.

25. Lara LAS, Lopes GP, Scalco SCP, Vale FBC, Rufino AC, Troncon JK, et al. Tratamento das disfunções sexuais no consultório do ginecologista. Rev. FEMINA. 2019; 47(2): 66-74. 
26. Alves ERP, Costa AM, Bezerra SMMS, Nakano AMS, Cavalcanti AMTS, Dias MD. Climatério: a intensidade dos sintomas e o desempenho sexual. Rev. SCIELO. 2015; 24(1): 64-71.

27. Barakat R, Oakley O, Kim H, Jin J, Ko CJ. Extra-gonadal sites of estrogen biosynthesis and function. BMB Rep. 2016; 49(9): 488-96.

28. Lucena CT, Soares MCS, Alves ERP, Ramos
DKR, Moura JP, Santos RC, et al. Percepção de mulheres no climatério sobre sua sexualidade. Rev. Univ. Vale do Rio Verde. 2014; 12(1): 28-37.

29. Reis LM, Moura AL, Haddad MCL, Vannuchi MTO, Smanioto FN. Influência do climatério no processo de trabalho de profissionais de um hospital universitário público. Cogitare Enferm. 2011; 16(2): 232-9. 